\title{
HSM Onkologie: Speerspitze einer Zentralisierung der ambulanten Versorgung?
}

\author{
Jürg Nadiga, \\ Günther Gruber ${ }^{b}$, \\ Markus Trutmann', \\ Marcus Schwöbel, \\ Stephan Eberhard, \\ Erika Ziltenerf, \\ Urs Saxer', \\ Andreas Lohri ${ }^{h}$
}

a Schweizerische Gesellschaft für Medizinische Onkologie (SGMO)

b Schweizerische Gesellschaft für Radioonkologie (SRO)

c foederatio medicorum chirurgicorum helvetica fmCh

d Kinderchirurgie

e Oncoreha.ch

f Dachverband Schweizerischer Patientenstellen (DVSP)

g Prof. Dr. iur. Rechtsanwalt, Zürich, VR Spital Männedorf AG, Rechtskonsulent KKA

h Leiter Onkologie Hämatologie, Medizinische Universitätsklinik, Kantonsspital Baselland, Standort Liestal

* In den Industrieländern basieren solche CCC auf 1020 Millionen Einwohnern. Sie integrieren Behandlung und Betreuung, Weiter- und Fortbildung, klinische Forschung und Grundlagenforschung. Wie die regionalen CCC mit weniger als einer Million Einwohner in der Schweiz dem Kriterium 4.3.4.3.b. der IV HSM «Internationale Konkurrenzfähigkeit» genügen wollen, bleibt offen.

Korrespondenz:

Dr. med. Jürg Nadig, MAE

Präsident der Schweizerischen

Gesellschaft für Medizinische Onkologie

Facharzt für Innere Medizin und Medizinische Onkologie Bannhaldenstrasse 7

CH-8180 Bülach

Tel. 0448627300

Fax 0448627301

juerg.nadig[at]hin.ch
Hochspezialisierte Medizin (HSM) Onkologie: ein Papiertiger oder Speerspitze einer zentralistischen Versorgungsstruktur?

Am 11. 12.2012 wurde der Bericht zur HSM Onkologie mit einer Vernehmlassungsfrist von nur fünf Wochen veröffentlicht (inkl. Feiertage), den eine Expertengruppe in den letzten drei Jahren ausgearbeitet hatte. Auf den ersten Blick scheint er ein Papiertiger zu sein: Die IVHSM (s. Kasten) kann über die Spitalliste lediglich etwa 5\% der onkologischen Versorgung regeln. Betroffen sind vor allem stationäre chirurgische Eingriffe. Auf den zweiten Blick wird das Konzept aber zur Speerspitze einer zentralistischen Versorgung im ambulanten Bereich. So umgesetzt, führt HSM Onkologie zu einem massiven Eingriff in die onkologische Versorgungsstruktur. Sieben sogenannte Comprehensive Cancer Centers (CCC)* legen parallel (!) in ihrer Region verbindlich Guidelines fest und lenken die Patientenströme (Einschränkung der freien Arzt- und Spitalwahl). Die CCC teilen die Schweiz kartellistisch unter sich auf. Das Angebot der übrigen onkologischen Leistungserbringer wird ohne Grund beschnitten: Sie dürfen keine modernen zielgerichteten medikamentösen Therapien mehr anbieten, obwohl sie das bisher unbeanstandet in guter Qualität seit Jahren gemacht haben (Einschränkung der Therapierfreiheit). Für Patientinnen und Patienten könnte das bedeuten, dass solche Therapien von den Krankenkassen nur noch übernommen werden, wenn sie diese von einem CCC erhalten. Statt eines einzigen nationalen Qualitätsregisters führt jedes der sieben Zentren ein eigenes Register. Alle nachgelagerten Institutionen müssen ihre Daten diesen Registern zuliefern. Die Datenhoheit liegt beim einzelnen CCC. Es wertet sie aus und publiziert sie. Die CCC bestimmen zudem, mit welchen Leistungserbringern sie zusammenarbeiten wollen.

\section{Meinungsumfrage}

In einer repräsentativen Umfrage der Schweizerischen Gesellschaft für Medizinische Onkologie (SGMO) lehnten 98,5\% der Abstimmenden das HSM-Onkologie-Konzept ab und unterstützten das Qualitätskonzept, das die Fachgesellschaft bereits ausgearbeitet hat. Die Rückmeldungen kamen aus allen Versorgungsstrukturen (Universitäts-, Kantons-, Schwerpunkt- und Privatspitäler, Gruppen- und Einzelpraxen) und allen Landesteilen.

Auch in den drei Bereichen Viszeralchirurgie, Kinderonkologie und Kinderchirurgie haben die Vor- schläge des HSM-Fachorgans Widerstand und Unwillen provoziert. Sind hier lediglich die Partialinteressen einiger Ewiggestriger tangiert oder bestehen methodische Mängel am Vorgehen des Fachorgans der HSM oder überschreitet es sogar seine Kompetenzen?

\section{Ziel der IV HSM}

Mit der Interkantonalen Vereinbarung zur Hochspezialisierten Medizin (IVHSM) delegieren die Kantone in diesem Bereich die Gestaltung ihrer Spitallisten an das HSM - Beschlussorgan (Art. 9.1 IVHSM). So soll über die Kantonsgrenzen hinweg die hochspezialisierte Medizin im stationären Bereich koordiniert werden. Diese Vereinbarung beschränkt sich auf Bereiche und Leistungen, die durch

- ihre Seltenheit,

- ihr hohes Innovationspotential,

- einen hohen personellen oder technischen Aufwand oder

komplexe Behandlungsverfahren

gekennzeichnet sind. Für die Zuordnung müssen mindestens drei der genannten Kriterien erfüllt sein, wobei aber immer das der Seltenheit vorliegen muss (Art. 1 IVHSM).

Vom Beschlussorgan angenommene und zugeteilte Leistungen heben abweichende kantonale Spi-

\section{IVHSM}

Die Kantone sind beauftragt, für den Bereich der hochspezialisierten Medizin (HSM) eine gemeinsame gesamtschweizerische Planung im Spitalbereich vorzunehmen (Art. 39, Abs. 2bis KVG). Für die Umsetzung dieses Gesetzesauftrages haben die Kantone per 1.1.2009 die Interkantonale Vereinbarung zur Hochspezialisierten Medizin (IVHSM) unterzeichnet und sich damit zur gemeinsamen Planung und Zuteilung von hochspezialisierten Leistungen verpflichtet. Für die hochspezialisierte Medizin gibt es somit anstelle von 26 kantonalen Planungen nur noch eine einzige, von allen Kantonen gemeinsam getragene Planung. Die IVHSM bildet die gesetzliche Grundlage für die Leistungszuteilung, legt die Entscheidungsprozesse der IVHSM Organe fest und definiert die Kriterien, welche ein Leistungsbereich erfüllen muss, um als hochspezialisierte Medizin im Sinne der IVHSM zu gelten. 
tallistenzulassungen auf (Art. 9.2 IVHSM). Das Beschlussorgan bestimmt die Bereiche der HSM, die einer schweizweiten Konzentration bedürfen. (Art. 3.3 IVHSM). Ein Fachorgan bereitet die Entscheidungen des Beschlussorgans vor. Die IVHSM legt fest, welche Voraussetzungen erfüllt sein müssen, dass eine Dienstleistung überhaupt zugeteilt werden kann (Art. 4.3 IVHSM):

a) Wirksamkeit

b) Nutzen

c) Technologisch-ökonomische Lebensdauer

d) Kosten der Leistung

Für den letzten Schritt, die Zuteilung an einzelne Leistungserbringer oder Institutionen, sind Qualität, Verfügbarkeit von hochqualifiziertem Personal und Teambildung, Verfügbarkeit der unterstützenden Disziplinen, Wirtschaftlichkeit und Weiterentwicklungspotential zu berücksichtigen. Die vom Fachorgan vorbereiteten Beschlüsse müssen fachbezogen und wissenschaftlich begründet sein (Art. 4.5 IVHSM).

\section{Bisherige Entscheide zur HSM}

In den letzten drei Jahren wurden zu bestimmten, genau umschriebenen Eingriffen Beschlüsse gefasst: Es wurde festgelegt, welche Voraussetzung für die Cochleaimplantate nötig seien und wo die Protonentherapie angeboten wird. Für die Neurochirurgie wurde die stereotaktische Chirurgie der anormalen Bewegungen und tiefe Hirnstimulation geregelt. Bei all diesen Bereichen ging es um Handlungskompetenzen in einem eng umschriebenen Gebiet mit hohem Innovationspotential. Diese Eingriffe sind als CHOP-Codes im SwissDRG-Tarif für stationäre Leistungen abgebildet. Nur eine scheinbare Ausnahme bildet die Versorgung der Schwerverletzten. Für ihre Behandlung braucht es aber eine hohe Handlungskompetenz verschiedener Disziplinen, die zeitnahe zusammenarbeiten und die unmittelbare Nachbehandlung koordinieren müssen.

\section{Krankheiten sind weder wirksam noch haben sie eine technologisch- ökonomische Lebensdauer}

Um in die Liste der HSM-Bereiche aufgenommen zu werden, ist Wirksamkeit, Nutzen, technologisch-ökonomische Lebensdauer und Kosten der Leistung zu berücksichtigen(Art. 4.4.1 IVHSM). Somit sind im Rahmen der IVHSM einzelne Leistungen/Eingriffe zu beurteilen und zuzuweisen. Es geht nicht um eine Globalvollmacht, Versorgungsgebiete aufzuteilen. Seltene Krankheiten per se erfüllen die Aufnahmekriterien der HSM nicht. Sie sind weder wirksam noch nützlich. Ihnen fehlt eine technologisch-ökonomische Lebensdauer. Es können nur einzelne Behandlungen oder Eingriffe, die diese Kriterien bei seltenen Krankheiten erfüllen, in die Liste der HSMBereiche aufgenommen werden. Alle bis jetzt geregelten Bereiche waren mit einer Handlungskompe- tenz verknüpft, die allenfalls koordiniert, zeitgleich von verschiedenen Spezialisten am gleichen Ort erbracht werden muss (Komplexe Behandlungsverfahren: Verbrennungen oder Schwerstverletzte) weshalb in Art. 4.3.4.2c IVHSM die ständige Verfügbarkeit der unterstützenden Disziplinen in gewissen Bereichen zu Recht eingefordert wird. Ein komplexes Behandlungsverfahren aus zeitlich gestaffelten, ambulant oder stationär erbrachten Routineeingriffen fällt nicht unter die IVHSM-Regelung, auch wenn die Krankheit selten ist. Es verlangt nach einem Behandlungskonzept mit verbindlicher Koordination der einzelnen Behandlungen, bspw. an einem (virtuellen) Tumorboard. Solche Behandlungskonzepte auszuarbeiten verlangt Wissenskompetenz. Expertise ist nicht an bauliche Strukturen, sondern an Personen gebunden.

Mit dem Bericht zur HSM Onkologie überschreitet das Fachorgan die gesetzlichen Rahmenbedingungen. Es regelt nicht mehr die Zuteilung einzelner stationärer Leistungen (Knochenmarktransplantation, Whippel Operation, Extremitätenperfusion ...), sondern schafft primär zentralistische Strukturen. Diese verursachen zwar durchaus Kosten und mehr unnötige Arbeit, kaum aber Nutzen. Selbst das Fachorgan der HSM Onkologie ist sich des Nutzens nicht sicher, schreibt es doch auf Seite 12, «dass durch eine schweizweite Koordination und Konzentration eine qualitative Verbesserung der Versorgung plausibel erscheint und voraussichtlich erreicht werden kann». Damit bestätigt es den Mangel an hierfür beweisenden Daten. Es geht lediglich von Annahmen aus. Dies widerspricht dem Grundsatz, dass Entscheide fachbezogen und wissenschaftlich sein müssten (Art. 4.5.3 IVHSM). Die Fachgesellschaft schlägt als zielführende Alternative fachgesellschaftseigene Qualitätsregister vor.

\section{Anträge sind fachbezogen und wissenschaftlich begründet}

Mit Qualitätsregister der Fachgesellschaft und Vollkosten liesse sich das Gesundheitswesen rationaler steuern als mit planwirtschaftlichen Eingriffen. DRG und neue Spitalfinanzierung sind ein erster Schritt zu mehr Kostentransparenz. Bezüglich Qualitätsdaten ist die Schweiz aber ein Entwicklungsland. Deshalb stützen sich die Entscheidungsträger oft auf Strukturkriterien ausländischer Empfehlungen. Es gibt aber einen Bereich, in dem Qualitätsdaten vorliegen: Im nationalen Kinderkrebsregister sind Behandlungsund Outcomedaten vorhanden. Was läge näher, als diese Qualitätsdaten für einen fachbezogenen und wissenschaftlich begründeten Zuteilungsentscheid $\mathrm{zu}$ verwenden (Art. 4.3.5 IVHSM)? Das Fachorgan begnügt sich aber mit dem Zählen von Eingriffen, die auf Strukturkriterien der Europäischen Gesellschaft für Pädiatrie beruhen! Scheute das Fachorgan die Kinderkrebsregisterdaten, weil sie eine sehr hohe Behandlungsqualität belegen? Bei einer hohen Qua- 
lität besteht kein Regelbedarf. Die Konzentration führt kaum zu einer Qualitätsverbesserung, da sie bereits sehr hoch ist. Gleichzeitig wurde verpasst, die ausländischen Strukturkriterien an unseren Outcomedaten zu validieren. Verschiedene Studien zeigen, dass eine Zentralisierung nicht mit einer besseren Qualität einhergehen muss.

\section{Verbessert die Zentralisierung die bereits vorhandene hohe Qualität?}

Vielleicht würden wir bei einem Fach wie der Neurochirurgie spontan sagen, die Konzentration auf wenige Orte sei zum Wohle der Kranken. Nun zeigt aber eine Untersuchung aus Norwegen, dass dem nicht zwangsläufig so ist. Das gleiche lässt sich für die Behandlung des Brustkrebses zeigen: Krebsregisterdaten aus Genf weisen für zentrale (Universitätsspital Genf) und dezentrale (niedergelassene Onkologen) Versorgungssysteme eine sehr hohe Behandlungsqualität aus. Stadien adaptiert ist der Outcome im Zentrum nicht besser als bei den niedergelassenen Onkologen. Ein gleiches Resultat zeigte eine kürzlich erschienene Studie zur Behandlung des M. Hodgkin in Deutschland: Die Behandlungsqualität an CCC ist gleich gut wie in kleineren Zentren und bei den niedergelassenen Onkologen. Ist die Qualität bereits sehr hoch, sind mit einer Zentralisierung kaum weitere Verbesserungen möglich.

\section{Transparente Prozesse und unabhängige Experten?}

Wenn nun höhere Behandlungsqualität in der Onkologie nicht an CCC gebunden ist, welche Interessen verstecken sich denn hinter einer Zentralisierung? Die von der HSM-Leitung beigezogenen Expertinnen und Experten sind nicht bekannt. Ihre Partikularinteressen werden nicht offengelegt. Somit fehlt dem Zentralisierungsprozess nicht nur die rechtliche Grundlage. Die methodischen Mängel und die fehlende Transparenz wecken Zweifel am vorgeschlagenen Konzept. Es bräuchte gute Gründe, den Ein- druck zu zerstreuen, ein kleiner Club von Interessensvertretern instrumentalisierten die HSM Onkologie, um unter dem Banner der Qualitätssicherung für sich selbst passende Regeln und eine Ausgrenzung der nichtuniversitären Onkologen zu schaffen.

\section{Wissen an Fachleute und nicht an Strukturen knüpfen: das Qualitätskonzept der Fachgesellschaften}

Die Fachgesellschaften der Radioonkologen und der Medizinischen Onkologen erarbeiteten zusammen mit der Onkologiepflege Schweiz ein Qualitätskonzept. Es beinhaltet interdisziplinäre Tumorboards und die Behandlung nach internationalen Guidelines. Zudem sollen Patientenpfade von den beteiligten Fachgesellschaften in der SAQM erarbeitet werden. Ein nationales Qualitätsregister der Fachgesellschaften basierend auf den Krebsregisterdaten ermöglicht Rückmeldungen der Behandlungsqualität an die einzelnen Leistungserbringer. Virtuelle (inter)nationale Kompetenzzentren für seltene Tumorkrankheiten mit interdisziplinären Tumorboards sollen den niederschwelligen Zugang zu Spezialistenwissen ermöglichen. Die im Swiss Cancer Network zusammengeschlossenen Fachärzte verpflichten sich schon heute, ihre Patientinnen und Patienten nach diesen Standards zu behandeln. Dieses Konzept braucht nicht 7 CCC, sondern ein Schweizerisches Krebs-Netzwerk, das die Aktivitäten auf verschiedenen Versorgungsebenen und in verschiedenen Bereichen (Vorsorge und Prävention, Behandlung und Betreuung Forschung) koordiniert. Den Fachgesellschaften und den Berufsorganisationen ist eine hohe Behandlungs- und Betreuungsqualität ein zentrales Anliegen. Die SAQM ist das geeignete Gefäss, diese Aktivitäten zu koordinieren. HSM Onkologie aber sollte Leistungen (CHOP-Codes im DRG-Tarif) für komplexe Eingriffe bei seltenen Krankheiten zuteilen, beispielsweise: Intraperitoneale Chemotherapie oder die Extremitätenperfusion. 